\title{
Malignancy-Associated Hemophagocytic Lymphohistiocytosis: An Aggressive Entity Which Requires Early Diagnosis
}

\author{
Nahla AM Hamed* \\ Professor of Clinical Hematology, Faculty of Medicine, Alexandria University, Egypt
}

Submission: March 07, 2019; Published: March 14, 2019

*Correspondence Author: Nahla AM Hamed, Professor of Clinical Hematology, Faculty of Medicine, Alexandria University, Egypt

Abstract

HLH comprises two main groups: malignancy-associated HLH (or M-HLH) and non-malignancy associated HLH. Treatment of HLH depends on the severity of the presentation as well as the underlying cause. M-HLH is an important subgroup of HLH because its prognosis is dismal. In addition, patients with M-HLH are already immunocompromised and addition of further cytotoxic therapy as a part of treatment for HLH is not an appealing option. Furthermore, it is difficult to abort therapy of the underlying malignancy and switch to therapy of HLH.

Abbreviations: HLH: Hemophagocytic Lymphohistiocytosis; MAS: Macrophage Activation Syndrome; M-HLH: Malignancy-Associated HLH

\section{Introduction}

The terms Hemophagocytic lymphohistiocytosis (HLH) and Lymphohistiocytic activation syndrome (LHAS) are equivalent [1]. HLH is not a malignant disorder. It is a highly stimulated defective severe inflammatory syndrome and tissue destruction resulting from an excessive immune activation and an absence of down-regulation of abnormally activated lymphocytes and macrophages [1].

\section{Epidemiology and classification of HLH}

The incidence of HLH is estimated to be approximately 1.2 cases per million individuals per year, but this is almost certainly an underestimate [2]. Recently, neoplasms have been recognized as triggers of HLH in children, with a prevalence of $8 \%$ to $11 \%$ [3].

\section{HLH is Classified into two Subgroups: Primary and Secondary}

Primary (genetic) HLH is a hereditary immune disorder [4] that may result from mutations in genes of cytolytic secretory pathway causing target cells apoptosis by perforin and granzymes. Primary HLH is divided into conditions in which HLH is the only disease manifestation (familial HLH, FHLH1-5), and those associated with other genetic causes in which HLH is one of several clinical manifestations [2].
Secondary HLH includes older children and adults who lack a family history or a known genetic cause for HLH [5]. Secondary HLH is the most common form seen in adults [6]. Extensive list of triggers is associated with secondary HLH [4].

\section{Secondary HLH develops as a complication of:}

i. Infection [4]: The most common infection associated with acquired HLH is Epstein-Barr virus [7]. Other implicated viruses are CMV, human immunodeficiency virus [5], respiratory syncytial virus, rotavirus, and adenovirus infections [7]. Bacterial, protozoal or mycotic infection can also induce HLH [5].

ii. Malignancy [4]: It can be divided into malignancy triggered forms, where malignancy itself leads to HLH, and the chemotherapy-associated HLH, where the antineoplastic treatment or its side effects trigger HLH [8]. These two etiologies might overlap during treatment of malignancies making their clear discrimination difficult [8]. HLH develops in the presence of malignancy, is known as malignancy-associated hemophagocytic syndrome (M-HLH) [8]. High grade lymphomas, mainly $\mathrm{T}$ cell and Natural killer non-Hodgkin lymphomas are the most common malignant causes of secondary HLH [1]. Other malignant causes are acute 


\section{Cancer Therapy \& Oncology International Journal}

leukemias, myelodysplastic syndromes, Langerhans cell histiocytosis, and histiocytic sarcoma [7]. Other commonly identified underlying malignancies included diffuse large $B$ cell lymphoma, Hodgkin lymphoma, CLL, CML, follicular lymphoma, splenic marginal B-cell lymphoma, PTLD, CMML. Solid tumors such as melanoma, breast cancer, testicular cancer, prostate cancer, squamous cell carcinoma of the neck, and pituitary mass were less frequently associated with HLH [9].

iii. Rheumatic diseases [4]: the macrophage activation syndrome (MAS) is a term commonly used to describe HLH secondary to rheumatological diseases [1]. MAS is most often found as a complication of systemic juvenile idiopathic arthritis, systemic lupus erythematosus, mixed connective tissue disease [7], polymyositis, vasculitis [5], polyarteritis nodosa, sarcoidosis, systemic sclerosis, Sjögren's syndrome and antiphospholipid syndrome [6].

iv. Drug hypersensitivity [4].

v. Spontaneous or iatrogenic immune suppression related HLH [5]. HLH has been observed in the setting of immune suppression after kidney and liver transplant and after allogeneic hematopoietic stem cell transplants [7].

vi. HLH can occur after prolonged periods of total parenteral nutrition that include soluble lipids. A condition known as fat overload syndrome [7].

vii. A case of HLH was reported in a neonate in association with mitochondrial respiratory chain disorder [7].

\section{Pathophysiology}

Three main pathways of the inflammatory response are central to HLH initiation and propagation: [1] hyperactivation of CD8+ $\mathrm{T}$ lymphocytes and macrophages, [2] proliferation and infiltration of these cells into organs and tissues, and [3] uncontrolled hypercytokinemia [9] (cytokine storm) produced by activated lymphocyte and macrophages [10]. Cytotoxic T cells and NK cells are unable to eliminate the overactivated macrophages leading to their further activation, and increased levels of interferon gamma, tumor necrosis factor alpha, soluble receptor of Interleukin (IL) 2 or CD25, IL-6 and IL-10. In addition, macrophages phagocytize host cells [1]. A thorough search for an underlying infection should be done [10] in any case of M-HLH or chemotherapy-associated HLH [8]. The hyperinflammatory state and disease manifestation in these cases seems to be the consequence of an inadequate reaction of the dysregulated immune system against common infectious agents [8].

\section{Clinical Presentation}

Patients are usually acutely and severely ill with multi-organ failure and without specific etiology despite prolonged hospital stay and evaluation. Common features include clinical deterioration, associated with persistent fever, high ferritin, hypertriglyceridemia, rash, lymphadenopathies, hepatosplenomegaly, neurologic symptoms, abnormal liver function tests, pancytopenia, coagulopathies, and sometimes acute kidney injury [1]. Differentiation between primary and secondary HLH is difficult due to indistinguishable clinical presentations, associated with excessive inflammatory cytokines and activation of macrophages [3].

The signs and symptoms, including prolonged fever and hepatosplenomegaly can be nonspecific [7]. Sepsis and shock can cause similar clinical features [10]. Decreased NK-cell activity and elevated sCD25 levels may help in differentiating HLH from sepsis. Typical clinical symptoms of HLH may resemble symptoms of malignant diseases or severe bacterial infections often occurring during antineoplastic treatment and subsequent immunodeficiency [8]. Fever, cytopenias, and hepatic dysfunction may all be explained by either malignant disease or the chemotherapy. That is why HLH diagnosis may be delayed in oncology patients [10]. HLH may precede, noted at the same time or even occurred after the diagnosis of malignancy. HLH is not diagnosed in patients at the time of remission [9]. The index of suspicion must be kept high, especially in the context of certain clinical disorders, such as immunosuppressed states, hematologic, infectious and rheumatologic diagnoses [7].

\section{Initial work up Includes}

Complete blood count, chemistry, liver and kidney function tests, coagulation studies (PT-INR, PTT, fibrinogen and d-dimer), serum triglycerides, ferritin, viral serologies and blood cultures. Bone marrow examination should be done to assess presence of hemophagocytes and to rule out lymphoproliferative disorders and leukemia [1]. About 1-10 hemophagocytes per 500 cells seen in bone marrow are considered positive [10]. All patients should also have cerebral spinal fluid studies, brain MRI and CT scan of the neck, chest, abdomen and pelvis to exclude any Central Nervous System pathology or other malignant etiology. Specialized immunologic testing should be done to patients highly suspected of having HLH: levels of soluble IL-2 receptor alpha; NK function or cell surface expression of CD107 alpha; soluble levels of the hemoglobin-haptoglobin scavenger receptor (sCD163), and perforin [1]. Studies to identify the underlying disorder should also be performed [4].

\section{Diagnosis}

Currently, HLH 2004 diagnostic criteria are followed worldwide in spite of the presence of a newer proposal put forth by HLH society in 2009 [6]. The 2004 diagnostic criteria for HLH requires 5 out of the following 8 clinical and pathological variables to be present: fever $>38.5^{\circ} \mathrm{C}$, splenomegaly, cytopenia in at least 2 lineages (hemoglobin $<9 \mathrm{~g} / \mathrm{dl}$; platelets $<100,000 /$ $\mu \mathrm{l}$; absolute neutrophil count $<1000 / \mu \mathrm{l}$ ), hypertriglyceridemia (fasting triglycerides $>265 \mathrm{mg} / \mathrm{dL}$ ) / hypofibrinogenemia (fibrinogen $<150 \mathrm{mg} / \mathrm{dL}$ ), hemophagocytosis on pathology examination (in bone marrow, spleen, lymph node, or liver), low/ absent NK cell activity, ferritin greater than $500 \mu \mathrm{g} / \mathrm{L}$ [11] 
and elevated soluble CD25 (soluble IL-2 receptor alpha) two standard deviations above age-adjusted laboratory-specific norms (> $2400 \mathrm{Ul} / \mathrm{ml}$ ) [1]. The two criteria (sIL2r $\alpha$ and natural killer-cell activity) have to be met among these 5 criteria [5].

The presence of either hemophagocytosis, or lymphohistiocytosis or hyperferritinemia alone may not be specific or sufficient to confirm or refute a diagnosis of HLH in adults [9]. Presence of hemophagocytosis in bone marrow alone is highly supportive but is neither diagnostic nor pathognomonic for HLH diagnosis [1]. Hyperferritinemia, mainly more than $10,000 \mathrm{~g} / \mathrm{dL}$ is of highly sensitive and specific value in diagnosing HLH [1]. The HLH-2004 criteria developed for children may be inadequate for accurately diagnosing HLH in adults [9]. Clinicians should never wait to meet these criteria to make the diagnosis and start the adequate treatment [1]. Strict use of the HLH-2004 criteria for diagnosis has pitfalls. Importantly, these criteria do not distinguish primary from secondary disease. Rather, they categorize a common phenotype characterized by toxic immune activation secondary to a range of disease processes, many of which have different treatments [3]. Identification of HLH in adults may require inclusion of additional diagnostic variables, especially variables that are easily obtained on routine laboratory or physical examination (9). Diagnostic criteria are usually used for clinical trials and are unlikely to be detected in every case of HLH [1].

\section{Treatment}

It is a fatal syndrome with high mortality rate [1] so treatment should never be delayed while awaiting some genetic tests or other specific immunologic testing [1]. Two considerations to be considered are suppression of hyperinflammation and targeting of the underlying disease if possible [7]. HLH specific treatment is based mainly on the HLH-94 protocol or clinical trials enrollment rather than treatment based on the HLH-2004 protocol [1]. High risk patients are those having HLH mutations, CNS disease and hematologic malignancies [1].

All primary HLH patients require allogeneic stem cell transplantation for cure as the problem is genetic [10]. The interferon-gamma inhibitor NI-0501 was recently approved breakthrough designation for the treatment of primary HLH [9].

In secondary HLH: Treatment of the underlying trigger(s) generally resolves immune dyregulation [3]. However, there are several obstacles to effective therapy in adult HLH. One of the major reasons is the presentation this entity at an advanced stage when it is not feasible to initiate effective therapy due to delay in its recognition [9]. M-HLH is an important subgroup of secondary HLH [8] because of its association with increased mortality [3]. In addition, patients with M-HLH are already immunocompromised and addition of further cytotoxic therapy is not an appealing option. Furthermore, it is difficult to abort therapy of the underlying malignancy and switch to therapy of

\section{HLH [9].}

In M-HLH, once symptoms are controlled, the disease-specific therapy for underlying malignancy should obviate the trigger and prevent recurrence [10]. Hemopoietic stem cell transplant is generally recommended in patients with recurrent/refractory disease, persistent NK-cell dysfunction, or CNS involvement [10]. The interferon-gamma inhibitor may prove to be a major milestone in the therapy of secondary and M-HLH. This agent does not cause myelosuppression and may be potentially given concomitant with anti-tumor therapy [9].

\section{Prognosis}

Secondary HLH in adults frequently manifests as an aggressive disease [5]. The earlier the diagnosis is made, and the quicker the treatment is given, the better will be the prognosis. Administration of the adequate treatment can dramatically increase the survival [1]. Approximately $56-70 \%$ of patients have a median overall survival of 36-230 days. The 3-year survival of M-HLH patients is $18-55 \%$ [12]. Out of all secondary triggers, M-HLH seems to have the worst outcome [10]. Mortality rates range from $8 \%$ in MAS complicating systemic juvenile idiopathic arthritis to $>80 \%$ in M-HLH [5]. Approximately, 50\% of secondary HLH patients die of irreversible multiorgan damage [10]. Prognostic factors associated with mortality include thrombocytopenia, high onset age, possible lymphoma (prognosis is better for B-cell than T-cell lymphoma-triggered HLH), increased ferritin levels, low fibrinogen level and EBV-DNA > 1,000 copies [12]. Infections, especially EBV infection and invasive fungal infection, may be the major factors in chemotherapy associated HLH patients [12]. Other predictive factors are CD3+ cells number, glycosylated ferritin concentration and 18F-2-fluoro-2-deoxy-D-glucose (FDG) bone marrow uptake on PET/CT [12].

\section{Recommendation}

Initiation of a comprehensive work-up for HLH to all patients who would have been diagnosed with hepatic or renal failure of unknown etiology, sudden onset multi-organ failure, culturenegative sepsis and encephalopathy of unknown etiology [9].

\section{Conclusion}

Early suspicious of HLH in oncology patients especially in those with fever, cytopenias, and hepatic dysfunction is important to avoid delay in diagnosis. These features may all be explained by either the disease or the chemotherapy.

\section{References}

1. EI Hachem G (2017) Hemophagocytic lymphohistiocytosis, a syndrome of excessive immune activation: Review of the literature. J Hematol Mult Myeloma 1(1): 1002.

2. Weitzman S (2011) Approach to hemophagocytic syndromes. Hematology Am Soc Hematol Educ Program 2011: 176-183.

3. Gurunathan A, Boucher AA, Mark M, Prus KM, O'Brien MM, et al. (2018) Limitations of HLH-2004 criteria in distinguishing malignancy- 
associated hemophagocytic lymphohistiocytosis. Pediatr Blood Cancer 65: e27400.

4. Morimoto A, Nakazawa Y, Ishii E (2016) Hemophagocytic lymphohistiocytosis: Pathogenesis, diagnosis, and management. Pediatrics International 58(9): 817-825.

5. Daver N, McClain K, Allen C, Parikh S, Otrock Z, et al. (2017) A consensus review on malignancy-associated hemophagocytic lymphohistiocytosis in adults. Cancer 123(17): 3229-3240.

6. Siddaiahgari SR, Agarwal S, Madukuri P, Moodahadu LS (2016) Hemophagocytic lymphohistiocytosis: A Review. J Blood Disord Transfus 7: 363.

7. Esteban YM, de Jong JLO, Tesher MS (2017) An overview of hemophagocytic lymphohistiocytosis. Pediatr Ann 46(8): e309-e313.

8. Strenger V, Merth G, Lackner H, Aberle SW, Kessler HH, et al. (2018) Malignancy and chemotherapy induced hemophagocytic lymphohistiocytosis in children and adolescents-a single centre experience of 20 years. Ann Hematol 97(6): 989-998.

9. Tamamyan G, Kantarjian H, Ning J, Jain P, Sasaki K, et al. (2016) Malignancy-associated HLH in adults: Relation to hemophagocytosis, characteristics, and outcomes. Cancer 122(18): 2857-2866.

10. Singh A, Dawman L, Seth R (2018) Malignancy associated hemophagocytic lymphohistiocytosis in children. J Can Res Ther 14(3): 559-562.

11. Delabie JMA, Tremblay-Lemay R, Calvente L, Gupta M, Tierens A, et al. (2018) S100B is a useful marker for the diagnosis of hemophagocytic lymphohistiocytosis. Blood 132: 3711.

12. Wang H, Xiong L, Tang W, Zhou Y, Li F (2017) A systematic review of malignancy-associated hemophagocytic lymphohistiocytosis that needs more attentions. Oncotarget 8(35): 59977-59985.

\section{Your next submission with Juniper Publishers will reach you the below assets}

- Quality Editorial service

- Swift Peer Review

- Reprints availability

- E-prints Service

- Manuscript Podcast for convenient understanding

- Global attainment for your research

- Manuscript accessibility in different formats ( Pdf, E-pub, Full Text, Audio)

- Unceasing customer service

Track the below URL for one-step submission https://juniperpublishers.com/online-submission.php 